\title{
Synthesis and Cyclic AMP Phosphodiesterase 4 Isoenzyme Inhibitory Activity of Heterocycle Condensed Purines
}

\author{
Hirokazu Suzuki, ${ }^{*, a}$ Manabu Yamamoto, ${ }^{d}$ Susumu Shimura, ${ }^{d}$ Ken-ichi Miуamoto, ${ }^{c}$ \\ Kenji Yамамото, ${ }^{b}$ and Hiroyuki SawanishI ${ }^{a}$ \\ ${ }^{a}$ Department of Synthetic Chemistry, Hokuriku University; and ${ }^{b}$ Department of Chemistry, Faculty of Pharmaceutical \\ Sciences, Hokuriku University; Ho-3 Kanagawa-machi, Kanazawa 920-1181, Japan: ${ }^{c}$ Department of Hospital Pharmacy, \\ School of Medicine; Kanazawa University; 13-1 Takara-machi, Kanazawa 920-8641, Japan: and ${ }^{d}$ Central Laboratory, \\ Lotte Co., Ltd.; 3-1-1 Numakage, Saitama 336-8601, Japan. $\quad$ Received January 31, 2002; accepted April 18, 2002
}

\begin{abstract}
To reverse the adverse reactions of alkylxanthines and to develop novel inhibitors of cyclic AMP phosphodiesterase 4 (PDE4), a series of heterocycle $[a]-,[b]-,[c, d]$-, and $[i]$-condensed purines were designed and synthesized. Although all compounds did not display PDE1 and PDE3 inhibitory activities, several heterocycle [i]-condensed purines strongly inhibited PDE4. Especially, $d l$-3,4-dipropyl-8-methyl-4,5,7,8-tetrahydro-1H-imidazo[2,1$i$ ]purin-5-one $(\mathrm{dl}-7 \mathrm{c})$ exhibited comparable PDE4 inhibitory activity $\left(\mathrm{IC}_{50}=1.9 \mu \mathrm{M}\right)$ to rolipram and denbufylline (DBF).
\end{abstract}

Key words PDE4 inhibitor; heterocycle condensed purine; tetrahydro-imidazo[2,1-i]purine

cAMP-phosphodiesterase 4 (PDE4) inhibitors have relaxation effects on bronchochial smooth muscle and anti-inflammatory activities, and have attracted attention as a remedy for asthmatic patients. ${ }^{1,2)}$ Our investigations on the structure-activity relationships of alkylxanthines as PDE4 inhibitors have shown that 1-, 3- and 7-substituents on the xanthine nucleus are important for PDE4 inhibitory activity. ${ }^{3-5}$ Although denbufylline (DBF) and XT-44, among compounds prepared by us, were effective against osteoporosis animal models, ${ }^{6,7)}$ two compounds caused emesis as an undesirable side effect, comparable to several prototype PDE4 inhibitors such as rolipram and its analogs. ${ }^{8)}$

Previous work in this series has shown that heterocycle condensed purines, namely tetrahydro- imidazo[2,1-i]purine (1) ${ }^{9)}$ and (2), ${ }^{10)}$ were effective for reducing the side effects of xanthines, and it became desirable to determine the effect of other condensed purines on PDE4 inhibitory activity.

As a continuation of studies on $[g, h]$-condensed purines (3) ${ }^{11)}$ we have designed and synthesized a series of heterocycles $[a]-(\mathbf{4}),[b]-(\mathbf{5}),[c, d]-(\mathbf{6})$, and $[i]$-condensed purines $(7,8)$ fixing the propyl group ${ }^{12)}$ as a substituent on the imidazole $N$ atom, and examined their in vitro effects on PDE isoenzymes.

\section{Chemistry}

[a]-Condensed purines (4) were prepared according to the method of Nagamatsu et al. ${ }^{13)}$ Treatment of 9 with propanal and sodium cyanoborohydride gave 5-(propylamino)imidazole (10), which was treated with benzoylisothiocyanate to give 11. Cyclization of $\mathbf{1 1}$ in aq. $\mathrm{NaOH}$ yielded 12. Successive $S$-methylation of $\mathbf{1 2}$, treatment of $\mathbf{1 3}$ with aminoalcohol, and ring closure of $\mathbf{1 4}$ with thionyl chloride gave the $[a]$-condensed purines $(\mathbf{4 a}, \mathbf{b}) . N$-Propylation of $\mathbf{4 a}, \mathbf{b}$ afforded $4 \mathbf{c}$ and $\mathbf{4 d}$, respectivery (Chart 3).

[b]-Condensed purines (5) were prepared according to the method of Ahn et al. ${ }^{14)}$ 7-Benzyl-1-propylxanthine (17), which was obtained from regioselective benzylation ${ }^{15)}$ of 5,6diamino-3-propyluracil (15) ${ }^{16)}$ and ring closure of 16 with diethoxymethyl acetate, was treated with excess phosphorus oxychloride to give 7-benzyl-2-chloropurine (18). By treat- ment of $\mathbf{1 8}$ with aminoalcohols and subsequent ring closure of 19a, $\mathbf{b}$ with thionyl chloride, the corresponding $[b]$-condensed purines $(\mathbf{2 0 a}, \mathbf{b})$ were obtained. Debenzylation of $\mathbf{2 0}$ by catalytic hydrogenation afforded the desired $\mathbf{5 a}, \mathbf{b}$, which were alkylated with $n$-propyl bromide in the presence of potassium carbonate to give $\mathbf{5 c}, \mathbf{d}$ (Chart 4).

$[c, d]$-Condensed purines (6) were prepared according to the method of Simo et al. ${ }^{17)}$ Catalytic hydrogenation of nitropyrimidines $(\mathbf{2 1} \mathbf{a}, \mathbf{b})^{18)}$ gave diamines $(\mathbf{2 2} \mathbf{a}, \mathbf{b})$, which were treated with ethyl orthoformate without purification to give the $[c, d]$-condensed purines $(\mathbf{6} \mathbf{a}, \mathbf{b})$, respectively. $N$-propylation of $\mathbf{6 a}, \mathbf{b}$ with $n$-propyl bromide in the presence of potassium carbonate yielded $\mathbf{6 c}, \mathbf{d}$ (Chart 5).

Substituted $[i]$-condensed purines $(\mathbf{7}, \mathbf{8})$ were prepared according to our reported method. ${ }^{9)}$ 6-(Hydroxyethylamino)purines $(d l-\mathbf{2 4}, d l-\mathbf{2 5}, d l-27$ and $d l-28)$ were obtained respec-

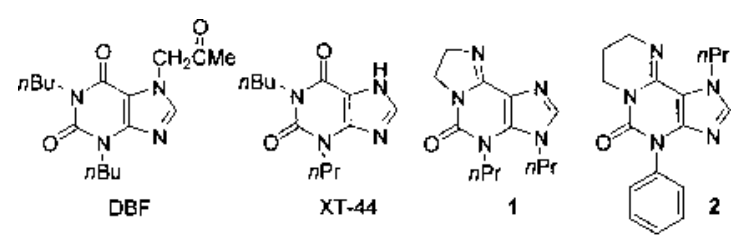

Chart 1

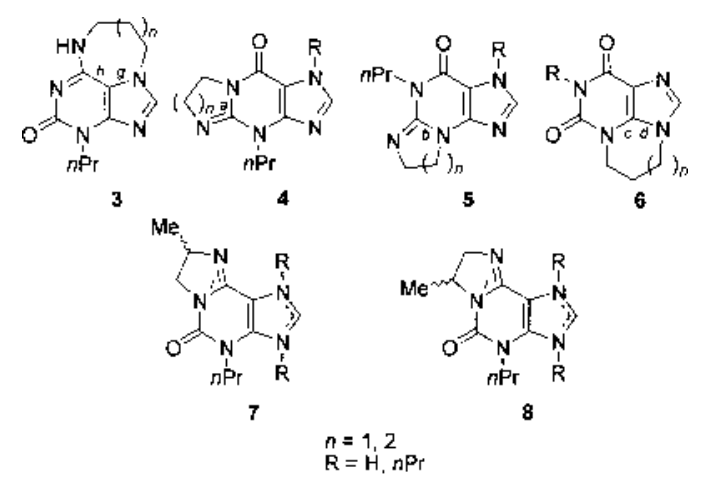

Chart 2 


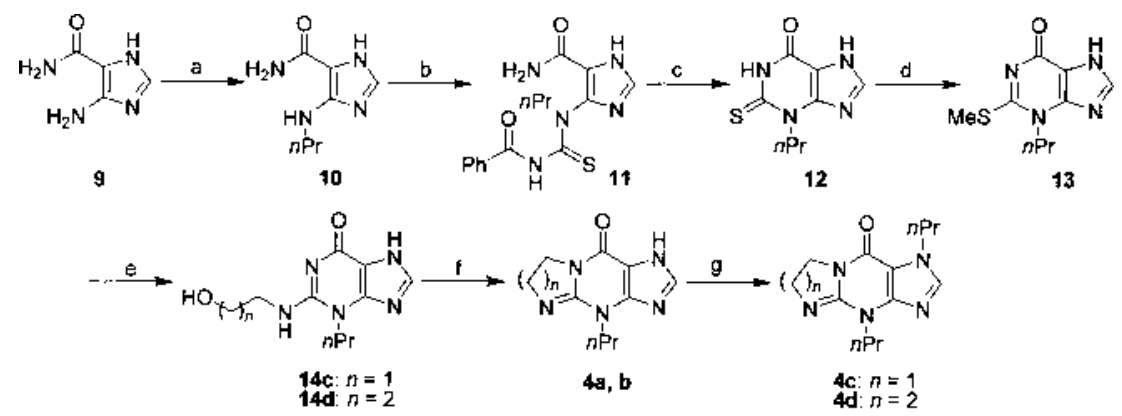

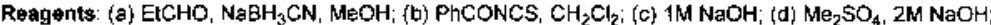

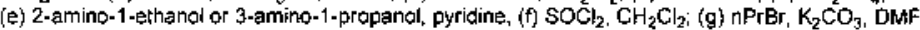

Chart 3
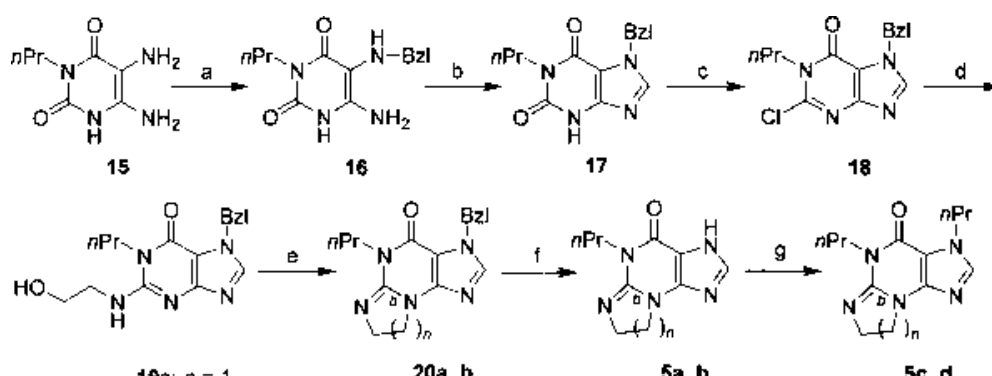

19a: $n=1$

$20 a, b$

$\mathbf{5 a}, \mathbf{b}$

5c, d

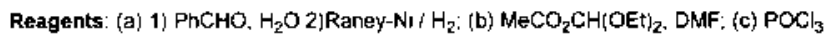

(d) 2-amino-1-ethanol or 3-arnino-1-propanol, pyridire; (e) $\mathrm{SOCl}_{2}, \mathrm{CH}_{2} \mathrm{Cl}$ 2 (f) $\mathrm{Pd}(\mathrm{OH})_{2}-\mathrm{C} / \mathrm{H}_{2}$. $\mathrm{MlOH}$ (g) $n \mathrm{P} r \mathrm{Br} . \mathrm{K}_{2} \mathrm{CO}_{3}$. $\mathrm{DWF}$

Chart 4

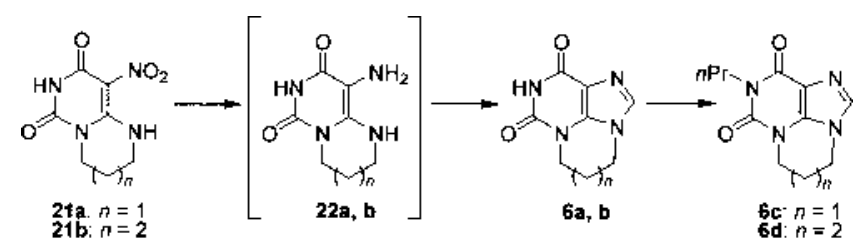

Reagents: (a) Pd-C. $\mathrm{H}_{2}, \mathrm{H}_{2} \mathrm{O}$; (b) HG(OEt)/3, DMF; (c) nPrBr, $\mathrm{K}_{2} \mathrm{CO}_{9}$, DMF

Chart 5
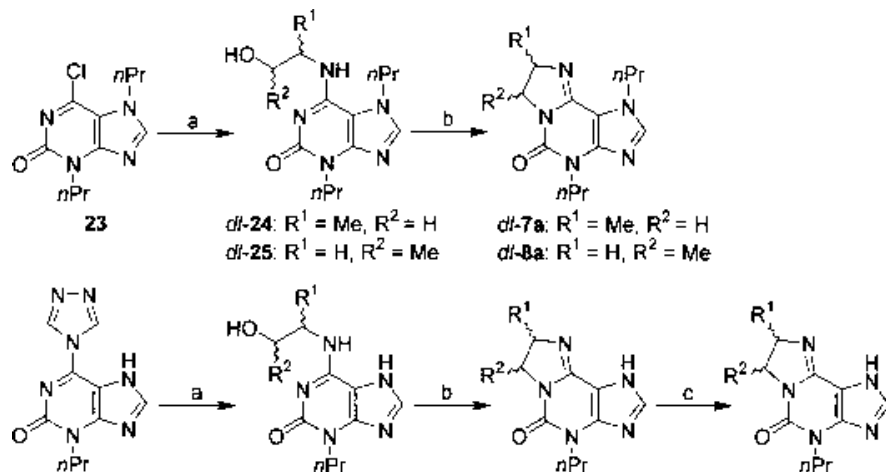

26 di-27: $R^{1}=M e, R^{2}=H \quad$ dit $b: R^{1}=M e, R^{2}=H \quad$ di-7c: $R^{1}=M e, R^{2}=H$

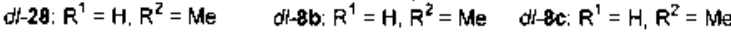

Reagents: (a) di-1-amino-2-propanel or ofl-2-amine-1-propanol, pyridine. (b) $\mathrm{MsCl}_{1} \mathrm{Et}_{3} \mathrm{~N}, \mathrm{CW} F$; (c) $\mathrm{nFrBr}, \mathrm{K}_{2} \mathrm{CO}_{3}$, DMF 
tively from reaction between 6-chloro-3,7-dipropylxanthine (23) or 3-propyl-6-(1,2,4-triazol-4-yl)purine (26) with each of $d l$-1-amino-2-propanol and $d l$-2-amino-1-propanol. $d l-24$, $d l-25, d l-27$ and $d l-28$ were reacted with methanesulfonyl chloride in the presence of triethylamine to give $d l-7 \mathbf{a}, d l-8 \mathbf{a}$, $d l-7 \mathbf{b}$ and $d l-8 \mathbf{b}$, respectively. $N$-Propylation of $d l-7 \mathbf{b}$ and $d l-$ $\mathbf{8 b}$ with $n$-propyl bromide in the presence potassium carbonate afforded $d l-7 \mathbf{c}$ and $d l-8 \mathbf{c}$, respectively (Chart 6).

\section{Results and Discussion}

The inhibitory activities of the heterocycle condensed purines (4-8) against PDE1 and PDE4 isoenzymes from guinea-pig brain and PDE3 from guinea-pig heart were measured according to the published methods. ${ }^{9)}$ The results are shown in Table 1 together with the PDE inhibitory activities of 1, 2, 3, XT-44, DBF and rolipram, reported already. ${ }^{19)}$

All newly prepared condensed purines showed no or only weak inhibitory activities against PDE1 and PDE3 isoenzymes. Against the PDE4 isoenzyme, the conclusions shown below were drawn.

1. The PDE4 inhibitory activities of heterocycle $[a]-(4)$, $[b]-(5),[c, d]-(\mathbf{6})$ and $[g, h]$-condensed purines (3) were weak. Compounds $4 \mathbf{a}, \mathbf{4 c}, \mathbf{5 a}$ and $\mathbf{5 b}$ inhibited selectively PDE4, but were weak compared with the $[i]$-condensed purines $(\mathbf{1}, \mathbf{2}, \mathbf{7}, \mathbf{8})$.

2. All of the heterocycle fused purines $(4,5,7,8)$, except $\mathbf{6}$, substituted with a propyl group at the $N$-position of the imidazole ring inhibited PDE4 more strongly than the corresponding unsubstituted compounds. $4 \mathbf{c}, 4 \mathbf{d} v s .4 \mathbf{a}, 4 \mathbf{b} ; \mathbf{5 c}, \mathbf{5 d}$ $v s .5 \mathbf{a}, \mathbf{5 b} ; d l-7 \mathbf{a}, d l-7 \mathbf{c} v s . d l-7 \mathbf{b} ; d l-8 \mathbf{a}, d l-8 \mathbf{c} v s . d l-\mathbf{8 b}$.

3. $N$-propyl substituted $[i]$-condensed purines $(d l-7 \mathbf{a}, \mathbf{c}$ and $d l-8 \mathbf{a}, \mathbf{c})$ having a methyl group at the 7- or 8-position, although they caused a decline in selectivity, exert more influence on the PDE4 inhibitory activities than $\mathbf{1}$ and $\mathbf{2}$.

In conclusion, $d l$-3,4-dipropyl-8-methyl-4,5,7,8-dihydro$1 H$-imidazo[2,1-i]purine-5-one (dl-7c) exhibited comparable PDE4 inhibitory activity to the known PDE4 inhibitors, rolipram and DBF. An earlier paper ${ }^{9)}$ has shown that the intramolecular interaction between the alkyl group at the 1-position and the carbonyl group at the 6-position of the xanthine skeleton may be important to elicit selectivity. This observation also suggests that the dihydroimidazole ring moiety of [i]-condensed purine may be important for PDE4 inhibitory activity. Currently, we are investigating the synthesis and PDE4 inhibitory activity of enantiomers of racemic heterocycle $[i]$-condensed purines $(\mathrm{dl}-\mathbf{7} \mathbf{\mathbf { a }}, \mathbf{c})$.

\section{Experimental}

Melting points were measured on a Yanagimoto micro melting point hot stage apparatus and are uncorrected. Infrared spectra (IR) were determined with a Horiba FT-720 spectrometer or a Hitachi 270-30 spectrometer. Mass spectra (MS) were measured with JEOL-DX300 instrument. Nuclear magnetic resonance spectra ( $\left.{ }^{1} \mathrm{H}-\mathrm{NMR}\right)$ were recorded on a JEOL EX 90A spectrometer. Chemical shifts are quoted in parts per million (ppm) with tetramethyl silane as an internal standard. Microanalyses were performed in the Micro Analytical Laboratory of this faculty. Yield and physicochemical data for the heterocycles $[a]-(\mathbf{4}),[b]-(\mathbf{5}),[c, d]-(\mathbf{6})$ and $[i]$-condensed purines $(\mathbf{7}$, 8) are summarized in Tables 2 and 3 , respectively.

5-Propylaminoimidazole-4-carboxamide (10) To a solution of 5aminoimidazole-4-carboxamide hydrochloride $9(1.0 \mathrm{~g}, 6.2 \mathrm{mmol})$ in $\mathrm{MeOH}$ $(10 \mathrm{ml})$ was added sodium cyanoborohydride $(0.31 \mathrm{~g}, 4.9 \mathrm{mmol})$ and propional $(0.43 \mathrm{~g}, 7.4 \mathrm{mmol})$, the reaction mixture was stirred for $24 \mathrm{~h}$ at room temperature (rt), then filtered, and evaporated in vacuo. The residue was chromatographed on silica gel using $\mathrm{CHCl}_{3}-\mathrm{MeOH}(6: 1)$ as an eluent to
Table 1. PDE Inhibitory Activities of Heterocycle Condensed Purines $(4-8)$

\begin{tabular}{|c|c|c|c|}
\hline \multirow{2}{*}{$\begin{array}{c}\text { Compd. } \\
\text { No. }\end{array}$} & \multicolumn{3}{|c|}{$\mathrm{IC}_{50}(\mu \mathrm{M})$} \\
\hline & PDE1 & PDE3 & PDE4 \\
\hline $4 a$ & $>100$ & $>100$ & $>100$ \\
\hline $4 b$ & $>100$ & $>100$ & $>100$ \\
\hline $4 c$ & $>100$ & $>100$ & 24 \\
\hline $4 d$ & $>100$ & $>100$ & 70 \\
\hline $5 a$ & $>100$ & $>100$ & $>100$ \\
\hline $5 b$ & $>100$ & $>100$ & $>100$ \\
\hline $5 c$ & $>100$ & $>100$ & 30 \\
\hline $5 d$ & $>100$ & $>100$ & 58 \\
\hline $6 a$ & $>100$ & $>100$ & $>100$ \\
\hline $6 b$ & $>100$ & $>100$ & $>100$ \\
\hline $6 c$ & $>100$ & $>100$ & $>100$ \\
\hline 6d & 92 & $>100$ & $>100$ \\
\hline$d l-7 \mathbf{a}$ & 41 & 94 & 4.7 \\
\hline$d l-7 \mathbf{b}$ & $>100$ & $>100$ & 88 \\
\hline$d l-7 \mathbf{c}$ & 35 & 92 & 1.9 \\
\hline$d l-8 \mathbf{a}$ & 77 & $>100$ & 19 \\
\hline$d l-\mathbf{8 b}$ & $>100$ & $>100$ & $>100$ \\
\hline$d l-\mathbf{8 c}$ & 98 & $>100$ & 15 \\
\hline 1 & $>100$ & 53 & 7.0 \\
\hline 2 & $>100$ & $>100$ & 5.7 \\
\hline $3 \mathbf{a}(n=1)$ & $>100$ & $>100$ & $>100$ \\
\hline $3 \mathbf{b}(n=2)$ & $>100$ & $>100$ & $>100$ \\
\hline XT-44 & $>100$ & $>100$ & 5.7 \\
\hline DBF & 78 & $>100$ & 1.5 \\
\hline Rolipram & $>100$ & $>100$ & 2.9 \\
\hline
\end{tabular}

Data are mean of three experiments.

give $10(0.66 \mathrm{~g}, 64 \%))$, which was recrystallized from $\mathrm{CHCl}_{3}-\mathrm{MeOH}$. mp $155-156{ }^{\circ} \mathrm{C} .{ }^{1} \mathrm{H}-\mathrm{NMR}\left(\mathrm{DMSO}-d_{6}\right) \delta: 0.88(3 \mathrm{H}, \mathrm{t}, J=7.1 \mathrm{~Hz}), 1.49(2 \mathrm{H}$, sext., $J=7.1 \mathrm{~Hz}), 3.14(2 \mathrm{H}, \mathrm{t}, J=7.1 \mathrm{~Hz}), 6.00(1 \mathrm{H}, \mathrm{brs}), 6.64(2 \mathrm{H}, \mathrm{br} \mathrm{s})$, $7.20(1 \mathrm{H}, \mathrm{s}), 11.57(1 \mathrm{H}, \mathrm{brs})$. IR $(\mathrm{KBr}) v_{\max }: 3413,3351,3302,1651$, $1631 \mathrm{~cm}^{-1}$. Anal. Calcd for $\mathrm{C}_{7} \mathrm{H}_{12} \mathrm{~N}_{4} \mathrm{O}: \mathrm{C}, 49.99 ; \mathrm{H}, 7.19 ; \mathrm{N}, 33.31$. Found: C, 50.13; H, 7.40; N, 33.47.

5-[ $N$-(Benzoylthiocarbamoyl)- $N$-propylamino]imidazole-4-carboxamide (11) To a suspension of $10(0.66 \mathrm{~g}, 3.9 \mathrm{mmol})$ in $\mathrm{CH}_{2} \mathrm{Cl}_{2}(15 \mathrm{ml})$ was added dropwise benzoylisothiocyanate $(0.77 \mathrm{~g}, 4.7 \mathrm{mmol})$, the reaction mixture was stirred for $6 \mathrm{~h}$ at rt. The precipitate was filtered and recrystallized from $\mathrm{MeOH}$ to give $11(0.88 \mathrm{~g}, 68 \%)$. mp $185-186^{\circ} \mathrm{C} .{ }^{1} \mathrm{H}-\mathrm{NMR}$ (DMSO$\left.d_{6}\right) \delta: 0.82(3 \mathrm{H}, \mathrm{t}, J=7.2 \mathrm{~Hz}), 1.59(2 \mathrm{H}$, sext., $J=7.2 \mathrm{~Hz}), 3.16(2 \mathrm{H}, \mathrm{t}, J=7.2$ $\mathrm{Hz}), 6.00(1 \mathrm{H}, \mathrm{br} \mathrm{s}), 6.64(2 \mathrm{H}, \mathrm{brs}), 7.52-8.00(8 \mathrm{H}, \mathrm{m}), 11.13(1 \mathrm{H}, \mathrm{brs}) . \mathrm{IR}$ (KBr) $v_{\max }: 3344,3284,3234,1658 \mathrm{~cm}^{-1}$. Anal. Calcd for $\mathrm{C}_{15} \mathrm{H}_{17} \mathrm{~N}_{5} \mathrm{O}_{2} \mathrm{~S}: \mathrm{C}$, 54.37; H, 5.17; N, 21.13. Found: C, 54.33; H, 5.40; N, 20.97.

3-Propyl-2-thioxanthine (12) A solution of $\mathbf{1 1}(2.7 \mathrm{~g}, 8.2 \mathrm{mmol})$ in $1 \mathrm{M}$ $\mathrm{NaOH}(30 \mathrm{ml})$ was heated to reflux for $3 \mathrm{~h}$. The reaction mixture was neutralized with hydrochloric acid and the precipitate was collected by filtration and recrystallized from $\mathrm{MeOH}$ to give $12(1.4 \mathrm{~g}, 79 \%) \mathrm{mp}>290{ }^{\circ} \mathrm{C} .{ }^{1} \mathrm{H}-$ NMR (DMSO- $\left.d_{6}\right) \delta: 0.91(3 \mathrm{H}, \mathrm{t}, J=7.2 \mathrm{~Hz}), 1.78(2 \mathrm{H}$, sext., $J=7.2 \mathrm{~Hz})$, $4.42(2 \mathrm{H}, \mathrm{t}, J=7.2 \mathrm{~Hz}), 8.16(1 \mathrm{H}, \mathrm{s}), 12.37(1 \mathrm{H}, \mathrm{br} \mathrm{s}) . \mathrm{IR}(\mathrm{KBr}) v_{\max }: 3471$, $1654,1620 \mathrm{~cm}^{-1}$. Anal. Calcd for $\mathrm{C}_{8} \mathrm{H}_{10} \mathrm{~N}_{4} \mathrm{OS}$ : C, 45.70; H, 4.79; N, 26.65 . Found: C, 45.88; H, 4.98; N, 26.69.

2-Methylthio-3-propylpurin-6-one (13) To a solution of $\mathbf{1 2}(0.64 \mathrm{~g}, 3.1$ $\mathrm{mmol})$ in $2 \mathrm{M} \mathrm{NaOH}(15 \mathrm{ml})$ was added dimethyl sulfate $(0.46 \mathrm{~g}, 3.7 \mathrm{mmol})$, and the reaction mixture was stirred for $1 \mathrm{~h}$ at $\mathrm{rt}$, then neutralized with acetic acid. The precipitate was collected by filtration and recrystallized from AcOEt-MeOH to give $13(0.41 \mathrm{~g}, 60 \%)$. mp $257-258^{\circ} \mathrm{C}$. ${ }^{1} \mathrm{H}-\mathrm{NMR}$ (DMSO- $\left.d_{6}\right) \delta: 0.92(3 \mathrm{H}, \mathrm{t}, J=7.2 \mathrm{~Hz}), 1.80(2 \mathrm{H}$, sext., $J=7.2 \mathrm{~Hz}), 2.56(3 \mathrm{H}$, s), $4.18(2 \mathrm{H}, \mathrm{t}, J=7.2 \mathrm{~Hz}), 8.10(1 \mathrm{H}, \mathrm{s}), 13.50(1 \mathrm{H}, \mathrm{brs})$. IR $(\mathrm{KBr}) v_{\max }$ : $3467,1630,1618 \mathrm{~cm}^{-1}$. Anal. Calcd for $\mathrm{C}_{9} \mathrm{H}_{12} \mathrm{~N}_{4} \mathrm{OS}: \mathrm{C}, 48.20 ; \mathrm{H}, 5.39 ; \mathrm{N}$, 24.98. Found: C, $48.31 ; \mathrm{H}, 5.18 ; \mathrm{N}, 24.83$.

2-(Hydroxyethylamino)-3-propylpurin-6-one (14a) and 2-(Hydroxypropylamino)-3-propylpurin-6-one (14b) A mixture of $\mathbf{1 3}(0.41 \mathrm{~g}, 1.8$ mmol) and aminoalcohol $(5 \mathrm{ml})$ in pyridine $(10 \mathrm{ml})$ was heated at reflux overnight and then concentrated in vacuo. The residue was recrystallized from AcOEt-MeOH to give $\mathbf{1 4}$.

14a: Yield: $83 \%$. mp $253-254{ }^{\circ} \mathrm{C} .{ }^{1} \mathrm{H}-\mathrm{NMR}\left(\mathrm{DMSO}_{6}\right) \delta$ : $0.88(3 \mathrm{H}, \mathrm{t}$, 
Table 2. Physicochemical Data for Heterocycle Condensed Purines $(4-8)$

\begin{tabular}{|c|c|c|c|c|c|c|c|c|}
\hline \multirow{2}{*}{ Compd. No. } & \multirow{2}{*}{$n$} & \multirow{2}{*}{ Yield $^{a)}(\%)$} & \multirow{2}{*}{$\mathrm{mp}\left({ }^{\circ} \mathrm{C}\right)$} & \multirow{2}{*}{ Recryst. solv. } & \multirow{2}{*}{ Formula } & \multicolumn{3}{|c|}{$\begin{array}{l}\text { Analysis (\%) } \\
\text { Calcd (Found) }\end{array}$} \\
\hline & & & & & & $\mathrm{C}$ & $\mathrm{H}$ & $\mathrm{N}$ \\
\hline $4 a$ & 2 & 70 & $256-257$ & AcOEt-MeOH & $\mathrm{C}_{10} \mathrm{H}_{13} \mathrm{~N}_{5} \mathrm{O}$ & $\begin{array}{c}54.78 \\
(54.99)\end{array}$ & $\begin{array}{l}5.98 \\
(6.05)\end{array}$ & $\begin{array}{c}31.94 \\
(31.97)\end{array}$ \\
\hline $4 b$ & 3 & 66 & $280-281$ & AcOEt-MeOH & $\mathrm{C}_{11} \mathrm{H}_{15} \mathrm{~N}_{5} \mathrm{O}$ & $\begin{array}{c}56.64 \\
(56.91)\end{array}$ & $\begin{array}{c}6.48 \\
(6.59)\end{array}$ & $\begin{array}{c}30.02 \\
(30.56)\end{array}$ \\
\hline $4 c$ & 2 & 63 & $108-109$ & Pet. Ether & $\mathrm{C}_{13} \mathrm{H}_{19} \mathrm{~N}_{5} \mathrm{O}$ & $\begin{array}{c}59.75 \\
(59.90)\end{array}$ & $\begin{array}{c}7.33 \\
(7.31)\end{array}$ & $\begin{array}{c}26.8 \\
(26.89)\end{array}$ \\
\hline $4 d$ & 3 & 62 & $105-106$ & Pet. Ether & $\mathrm{C}_{14} \mathrm{H}_{21} \mathrm{~N}_{5} \mathrm{O}$ & $\begin{array}{c}61.07 \\
(61.21)\end{array}$ & $\begin{array}{c}7.69 \\
(7.78)\end{array}$ & $\begin{array}{c}25.43 \\
(25.53)\end{array}$ \\
\hline $5 \mathbf{a}$ & 2 & 87 & $242-243$ & AcOEt-MeOH & $\mathrm{C}_{10} \mathrm{H}_{13} \mathrm{~N}_{5} \mathrm{O}$ & $\begin{array}{c}54.78 \\
(54.53)\end{array}$ & $\begin{array}{l}5.98 \\
(6.04)\end{array}$ & $\begin{array}{c}31.94 \\
(31.77)\end{array}$ \\
\hline $5 b$ & 3 & 72 & $261-262$ & AcOEt-MeOH & $\mathrm{C}_{11} \mathrm{H}_{15} \mathrm{~N}_{5} \mathrm{O}$ & $\begin{array}{c}56.64 \\
(56.45)\end{array}$ & $\begin{array}{c}6.48 \\
(6.47)\end{array}$ & $\begin{array}{c}30.02 \\
(29.97)\end{array}$ \\
\hline $5 c$ & 2 & 63 & $60-61$ & Pet. Ether & $\mathrm{C}_{13} \mathrm{H}_{19} \mathrm{~N}_{5} \mathrm{O}$ & $\begin{array}{c}59.75 \\
(57.91)\end{array}$ & $\begin{array}{c}7.33 \\
(7.32)\end{array}$ & $\begin{array}{c}26.80 \\
(25.85)\end{array}$ \\
\hline $5 d$ & 3 & 30 & Oil & - & $\mathrm{C}_{14} \mathrm{H}_{21} \mathrm{~N}_{5} \mathrm{O}$ & & $\begin{array}{l}275.1746 \\
257.1752^{b)}\end{array}$ & \\
\hline $6 \mathbf{a}$ & 3 & 51 & $>290$ & $\mathrm{EtOH}$ & $\mathrm{C}_{8} \mathrm{H}_{8} \mathrm{~N}_{4} \mathrm{O}_{2}$ & $\begin{array}{c}50.00 \\
(49.81)\end{array}$ & $\begin{array}{c}4.20 \\
(4.18)\end{array}$ & $\begin{array}{c}29.15 \\
(28.99)\end{array}$ \\
\hline $6 b$ & 4 & 56 & $>290$ & $\mathrm{EtOH}$ & $\mathrm{C}_{9} \mathrm{H}_{10} \mathrm{~N}_{4} \mathrm{O}_{2}$ & $\begin{array}{c}52.42 \\
(52.33)\end{array}$ & $\begin{array}{c}4.89 \\
(4.95)\end{array}$ & $\begin{array}{c}27.17 \\
(26.95)\end{array}$ \\
\hline $6 c$ & 3 & 45 & $248-249$ & AcOEt-MeOH & $\mathrm{C}_{11} \mathrm{H}_{14} \mathrm{~N}_{4} \mathrm{O}_{2}$ & $\begin{array}{c}56.40 \\
(56.30)\end{array}$ & $\begin{array}{c}6.02 \\
(5.95)\end{array}$ & $\begin{array}{c}23.92 \\
(23.88)\end{array}$ \\
\hline 6d & 4 & 51 & $204-205$ & AcOEt-MeOH & $\mathrm{C}_{12} \mathrm{H}_{16} \mathrm{~N}_{4} \mathrm{O}_{2}$ & $\begin{array}{c}58.08 \\
(57.92)\end{array}$ & $\begin{array}{c}6.50 \\
(6.34)\end{array}$ & $\begin{array}{c}22.57 \\
(22.56)\end{array}$ \\
\hline$d l-7 \mathbf{a}$ & - & 83 & $128-129$ & Pet. Ether & $\mathrm{C}_{14} \mathrm{H}_{21} \mathrm{~N}_{5} \mathrm{O}$ & $\begin{array}{c}61.07 \\
(61.33)\end{array}$ & $\begin{array}{c}7.69 \\
(7.72)\end{array}$ & $\begin{array}{c}25.43 \\
(25.38)\end{array}$ \\
\hline$d l-7 \mathbf{b}$ & - & 71 & $248-249$ & AcOEt-MeOH & $\mathrm{C}_{11} \mathrm{H}_{15} \mathrm{~N}_{5} \mathrm{O}$ & $\begin{array}{c}56.64 \\
(56.57)\end{array}$ & $\begin{array}{c}6.48 \\
(6.51)\end{array}$ & $\begin{array}{c}30.02 \\
(29.97)\end{array}$ \\
\hline$d l-7 \mathrm{c}$ & - & 85 & $111-112$ & Pet. Ether & $\mathrm{C}_{14} \mathrm{H}_{21} \mathrm{~N}_{5} \mathrm{O}$ & $\begin{array}{c}61.07 \\
(61.33)\end{array}$ & $\begin{array}{c}7.69 \\
(7.84)\end{array}$ & $\begin{array}{c}25.43 \\
(25.50)\end{array}$ \\
\hline$d l-8 \mathbf{a}$ & - & 74 & Oil & - & $\mathrm{C}_{14} \mathrm{H}_{21} \mathrm{~N}_{5} \mathrm{O}$ & & $\begin{array}{l}275.1746 \\
275.1744^{b)}\end{array}$ & \\
\hline$d l-\mathbf{8 b}$ & - & 77 & $272-273$ & AcOEt-MeOH & $\mathrm{C}_{11} \mathrm{H}_{15} \mathrm{~N}_{5} \mathrm{O}$ & $\begin{array}{c}56.64 \\
(56.71)\end{array}$ & $\begin{array}{c}6.48 \\
(6.66)\end{array}$ & $\begin{array}{c}30.02 \\
(29.94)\end{array}$ \\
\hline$d l-8 \mathrm{c}$ & - & 83 & Oil & - & $\mathrm{C}_{14} \mathrm{H}_{21} \mathrm{~N}_{5} \mathrm{O}$ & & $\begin{array}{l}275.1746 \\
275.1749^{b)}\end{array}$ & \\
\hline
\end{tabular}

a) Yield of final step and for purified product. b) High resolution MS spectra data.

$J=7.2 \mathrm{~Hz}), 1.70(2 \mathrm{H}$, sext., $J=7.2 \mathrm{~Hz}), 3.38-3.62(4 \mathrm{H}, \mathrm{m}), 4.05(2 \mathrm{H}, \mathrm{t}$, $J=7.2 \mathrm{~Hz}), 4.90(1 \mathrm{H}, \mathrm{brs}), 6.97(1 \mathrm{H}, \mathrm{br} \mathrm{s}), 7.84(1 \mathrm{H}, \mathrm{s}), 12.98(1 \mathrm{H}, \mathrm{br} \mathrm{s})$. IR (KBr) $v_{\max }: 3448,3288,3164,1630,1606 \mathrm{~cm}^{-1}$. Anal. Calcd for $\mathrm{C}_{10} \mathrm{H}_{15} \mathrm{~N}_{5} \mathrm{O}_{2}$ : C, 50.62; H, 6.37; N, 29.52. Found: C, 50.43; H, 6.40; N, 29.67.

14b: Yield: $71 \%$. mp $194-195^{\circ} \mathrm{C} .{ }^{1} \mathrm{H}-\mathrm{NMR}$ (DMSO- $\left.d_{6}\right) \delta: 0.88(3 \mathrm{H}, \mathrm{t}$, $J=7.2 \mathrm{~Hz}), 1.45-1.83(4 \mathrm{H}, \mathrm{m}), 4.04(2 \mathrm{H}, \mathrm{t}, J=7.3 \mathrm{~Hz}), 4.68(1 \mathrm{H}, \mathrm{br} \mathrm{s}), 6.97$ $(1 \mathrm{H}, \mathrm{brs}), 7.84(1 \mathrm{H}, \mathrm{s}), 12.69(1 \mathrm{H}, \mathrm{br} \mathrm{s})$. IR $(\mathrm{KBr}) v_{\max }: 3421,3282,3337$, $1681 \mathrm{~cm}^{-1}$. Anal. Calcd for $\mathrm{C}_{11} \mathrm{H}_{17} \mathrm{~N}_{5} \mathrm{O}_{2}: \mathrm{C}, 52.58 ; \mathrm{H}, 6.82 ; \mathrm{N}, 27.87$. Found: C, 52.73; H, 6.70; N, 27.77.

4-Propyl-4,5,6,7-tetrahydro-1 $H$-imidazo[1,2- $a]$ purin-9-one (4a) and 4Propyl-4,5,6,7-tetrahydro-1 $H$-pyrimido[1,2-a]purin-10-one (4b) A mixture of $14(0.63 \mathrm{mmol})$ and $\mathrm{SOCl}_{2}(0.23 \mathrm{~g}, 1.9 \mathrm{mmol})$ in $\mathrm{CH}_{2} \mathrm{Cl}_{2}(30 \mathrm{ml})$ was stirred for $24 \mathrm{~h}$ at $\mathrm{rt}$. The reaction mixture was evaporated in vacuo, and the residue was chromatographed on silica gel using $\mathrm{CHCl}_{3}-\mathrm{MeOH}(3: 1)$ as an eluent to give 4, which was recrystallized from AcOEt-MeOH.

1,4-Dipropyl-4,5,6,7-tetrahydro-1 $H$-imidazo[1,2-a]purin-9-one (4c) and 1,4-Dipropyl-4,5,6,7-tetrahydro- $1 H$-pyrimido[1,2-a]purin-10-one (4d) To a mixture of $4 \mathbf{a}$ or $4 \mathbf{b}(2.0 \mathrm{mmol})$ and anhydrous $\mathrm{K}_{2} \mathrm{CO}_{3}(0.41 \mathrm{~g}$, $2.9 \mathrm{mmol}$ ) in $N, N$-dimethylformamide (DMF, $10 \mathrm{ml}$ ) was added propyl bromide $(0.37 \mathrm{~g}, 3.0 \mathrm{mmol})$, and the mixture was stirred at $\mathrm{rt}$ for $10 \mathrm{~h}$ then concentrated in vacuo. The residue was chromatographed on silica gel using $\mathrm{CHCl}_{3}-\mathrm{MeOH}(20: 1)$ as an eluent to give $\mathbf{4 c}$ and $\mathbf{4 d}$, which was recrystallized from petroleum ether.

6-Amino-5-(benzylamino)-3-propyluracil (16) A mixture of $\mathbf{1 5}(3.7 \mathrm{~g}$, $20 \mathrm{mmol})$ and benzaldehyde $(3.2 \mathrm{~g}, 30 \mathrm{mmol})$ in $\mathrm{H}_{2} \mathrm{O}(150 \mathrm{ml})$ was stirred for $2 \mathrm{~h}$ at rt. The solution was cooled and the benzylidene was separated from the solution. The benzylidene was then hydrogenated over Raney-Ni
$(2.0 \mathrm{~g})$ in $1 \mathrm{~m} \mathrm{NaOH}(100 \mathrm{ml})$ at 3 atom for $12 \mathrm{~h}$. After removal of catalyst and concentration the filtrate, then adjusting the $\mathrm{pH}$ to 6 with acetic acid, the crude product was recrystallized from EtOH to give $\mathbf{1 6}(4.10 \mathrm{~g}, 75 \%)$. mp $210-211^{\circ} \mathrm{C} .{ }^{1} \mathrm{H}-\mathrm{NMR}$ (DMSO- $\left.d_{6}\right) \delta: 0.81(3 \mathrm{H}, \mathrm{t}, J=7.3 \mathrm{~Hz}), 1.48(2 \mathrm{H}$, sext., $J=7.3 \mathrm{~Hz}), 3.65(2 \mathrm{H}, \mathrm{t}, J=7.3 \mathrm{~Hz}), 3.82(2 \mathrm{H}, \mathrm{s}), 5.73(1 \mathrm{H}, \mathrm{brs})$, $7.20-7.38(7 \mathrm{H}, \mathrm{s}), 10.19(1 \mathrm{H}, \mathrm{br})$. IR (KBr) $v_{\max }: 3332,3268,3196,1698$, $1662 \mathrm{~cm}^{-1}$. Anal. Calcd for $\mathrm{C}_{14} \mathrm{H}_{18} \mathrm{~N}_{4} \mathrm{O}_{2}: \mathrm{C}, 61.30 ; \mathrm{H}, 6.61 ; \mathrm{N}, 20.42$. Found: C, 61.46; H, 6.60; N, 20.26 .

7-Benzyl-1-propylxanthine (17) A mixture of $\mathbf{1 6}(2.7 \mathrm{~g}, 9.9 \mathrm{mmol})$ and diethoxymethyl acetate $(6.4 \mathrm{~g}, 39 \mathrm{mmol})$ in DMF $(15 \mathrm{ml})$ was stirred for $5 \mathrm{~h}$ at $80^{\circ} \mathrm{C}$. The reaction mixture was concentrated in vacuo and the residue was recrystallized from $\mathrm{MeOH}$ to give $17(1.7 \mathrm{~g}, 61 \%)$. mp $177-178^{\circ} \mathrm{C}$. ${ }^{1} \mathrm{H}-\mathrm{NMR}$ (DMSO- $\left.d_{6}\right) \delta: 0.84(3 \mathrm{H}, \mathrm{t}, J=7.3 \mathrm{~Hz}), 1.52(2 \mathrm{H}$, sext., $J=7.3 \mathrm{~Hz})$, $3.76(2 \mathrm{H}, \mathrm{t}, J=7.3 \mathrm{~Hz}), 5.45(2 \mathrm{H}, \mathrm{s}), 7.33(5 \mathrm{H}, \mathrm{s}), 8.15(1 \mathrm{H}, \mathrm{s}), 11.86(1 \mathrm{H}$, br s). IR (KBr) $v_{\max }: 3504,1709,1657 \mathrm{~cm}^{-1}$. Anal. Calcd for $\mathrm{C}_{15} \mathrm{H}_{16} \mathrm{~N}_{4} \mathrm{O}_{2}: \mathrm{C}$, 63.37; H, 5.67; N, 19.71. Found: C, 63.41; H, 5.77; N, 19.67.

7-Benzyl-2-chloro-1-propylxanthine (18) A mixture of $\mathbf{1 7}(1.0 \mathrm{~g}, 3.7$ mmol) and $\mathrm{POCl}_{3}(20 \mathrm{ml})$ was refluxed for $5 \mathrm{~h}$. The reaction mixture was concentrated in vacuo and the residue was partitioned between $\mathrm{CHCl}_{3}$ and sat. $\mathrm{NaHCO}_{3}$ solution and the organic layer was washed with brine, dried and concentrated. The crude product was chromatographed on silica gel using $\mathrm{CHCl}_{3}-\mathrm{MeOH}(20: 1)$ as an eluent to give $18(0.44 \mathrm{~g}, 40 \%)$, which was recrystallized from MeOH. mp $142-143^{\circ} \mathrm{C} .{ }^{1} \mathrm{H}-\mathrm{NMR}$ (DMSO- $d_{6}$ ) $\delta$ : $1.01(3 \mathrm{H}, \mathrm{t}, J=7.5 \mathrm{~Hz}), 1.80(2 \mathrm{H}$, sext., $J=7.5 \mathrm{~Hz}), 4.24(2 \mathrm{H}, \mathrm{t}, J=7.5 \mathrm{~Hz})$, $5.61(2 \mathrm{H}, \mathrm{s}), 7.59(5 \mathrm{H}, \mathrm{s}), 7.84(1 \mathrm{H}, \mathrm{s})$. IR (KBr) $v_{\mathrm{max}}: 1707 \mathrm{~cm}^{-1}$. MS $\mathrm{m} / \mathrm{z}$ : $302\left(\mathrm{M}^{+}\right), 304\left(\mathrm{M}^{+}+2\right)$. Anal. Calcd for $\mathrm{C}_{15} \mathrm{H}_{15} \mathrm{~N}_{4} \mathrm{OCl}$ : C, 59.51; H, 4.99; N, 18.51. Found: C, 59.52; H, 5.07; N, 18.53 .

7-Benzyl-2-(hydroxyethylamino)-1-propylpurin-6-one (19a) and 7- 
Table 3. ${ }^{1}$ H-NMR Data of Heterocycle Condensed Purines (4-8)

\begin{tabular}{|c|c|c|}
\hline Compd. No. & $\operatorname{IR}(\mathrm{KBr}) \mathrm{cm}^{-1}$ & ${ }^{1} \mathrm{H}-\mathrm{NMR}$ \\
\hline $4 a^{a)}$ & $3458,1722,1639$ & $0.95(3 \mathrm{H}, \mathrm{t}, J=7.2 \mathrm{~Hz}), 1.77(2 \mathrm{H}, \mathrm{sext} ., J=7.2 \mathrm{~Hz}), 3.82-4.36(6 \mathrm{H}, \mathrm{m}), 8.24(1 \mathrm{H}, \mathrm{s}), 12.19(1 \mathrm{H}, \mathrm{br} \mathrm{s})$ \\
\hline $4 b^{a)}$ & $3471,1716,1597$ & $\begin{array}{l}0.93(3 \mathrm{H}, \mathrm{t}, J=7.2 \mathrm{~Hz}), 1.45(2 \mathrm{H}, \text { sext., } J=7.2 \mathrm{~Hz}), 1.96(2 \mathrm{H}, \text { quint., } J=5.6 \mathrm{~Hz}), 3.47(2 \mathrm{H}, \mathrm{t}, J=5.6 \mathrm{~Hz}) \\
3.95(2 \mathrm{H}, \mathrm{t}, J=5.6 \mathrm{~Hz}), 4.18(2 \mathrm{H}, \mathrm{t}, J=7.2 \mathrm{~Hz}), 8.13(1 \mathrm{H}, \mathrm{s}), 12.03(1 \mathrm{H}, \mathrm{br} \mathrm{s})\end{array}$ \\
\hline $4 c^{b)}$ & 1682,1637 & $0.94(3 \mathrm{H}, \mathrm{t}, J=7.3 \mathrm{~Hz}), 0.98(3 \mathrm{H}, \mathrm{t}, J=7.3 \mathrm{~Hz}), 1.64-2.12(4 \mathrm{H}, \mathrm{m}), 3.86-4.25(8 \mathrm{H}, \mathrm{m}), 7.39(1 \mathrm{H}, \mathrm{s})$ \\
\hline $\mathbf{4 d}^{b)}$ & 1676,1633 & $\begin{array}{l}0.94(3 \mathrm{H}, \mathrm{t}, J=7.3 \mathrm{~Hz}), 0.96(3 \mathrm{H}, \mathrm{t}, J=7.3 \mathrm{~Hz}), 1.49-2.01(6 \mathrm{H}, \mathrm{m}), 3.53(2 \mathrm{H}, \mathrm{t}, J=5.8 \mathrm{~Hz}), 3.84-4.25 \\
(6 \mathrm{H}, \mathrm{m}), 7.39(1 \mathrm{H}, \mathrm{s})\end{array}$ \\
\hline $\mathbf{5 a}^{a)}$ & $3471,1685,1631$ & $\begin{array}{l}0.98(3 \mathrm{H}, \mathrm{t}, J=7.5 \mathrm{~Hz}), 1.85(2 \mathrm{H}, \text { sext., } J=7.5 \mathrm{~Hz}), 3.97(2 \mathrm{H}, \mathrm{t}, J=7.5 \mathrm{~Hz}), 4.08(2 \mathrm{H}, \mathrm{t}, J=5.3 \mathrm{~Hz}), 4.10 \\
(2 \mathrm{H}, \mathrm{t}, J=5.3 \mathrm{~Hz}), 7.64(1 \mathrm{H}, \mathrm{s})\end{array}$ \\
\hline $\mathbf{5 b}^{a)}$ & $3496,1676,1635$ & $\begin{array}{l}0.95(3 \mathrm{H}, \mathrm{t}, J=7.3 \mathrm{~Hz}), 1.69(2 \mathrm{H}, \text { sext., } J=7.3 \mathrm{~Hz}), 1.94(2 \mathrm{H}, \text { quint., } J=5.7 \mathrm{~Hz}), 3.60(2 \mathrm{H}, \mathrm{t}, J=5.7 \mathrm{~Hz}) \\
4.01(2 \mathrm{H}, \mathrm{t}, J=5.7 \mathrm{~Hz}), 4.03(2 \mathrm{H}, \mathrm{t}, J=7.3 \mathrm{~Hz}), 7.62(1 \mathrm{H}, \mathrm{s})\end{array}$ \\
\hline $5 c^{b)}$ & 1670,1623 & $\begin{array}{l}0.93(3 \mathrm{H}, \mathrm{t}, J=7.5 \mathrm{~Hz}), 0.96(3 \mathrm{H}, \mathrm{t}, J=7.5 \mathrm{~Hz}), 1.71(2 \mathrm{H}, \text { sext., } J=7.5 \mathrm{~Hz}), 1.71(2 \mathrm{H}, \text { sext., } J=7.5 \mathrm{~Hz}) \\
3.75-4.26(8 \mathrm{H}, \mathrm{m}), 7.38(1 \mathrm{H}, \mathrm{s})\end{array}$ \\
\hline $5 \mathbf{d}^{b)}$ & 1687,1633 & $\begin{array}{l}0.94(6 \mathrm{H}, \mathrm{t}, J=7.3 \mathrm{~Hz}), 1.46-1.85(4 \mathrm{H}, \mathrm{m}), 3.58(2 \mathrm{H}, \mathrm{t}, J=5.7 \mathrm{~Hz}), 3.93(2 \mathrm{H}, \mathrm{t}, J=5.7 \mathrm{~Hz}), 4.04(2 \mathrm{H}, \mathrm{t} \\
J=7.3 \mathrm{~Hz}), 4.20(2 \mathrm{H}, \mathrm{t}, J=7.3 \mathrm{~Hz}), 7.37(1 \mathrm{H}, \mathrm{s})\end{array}$ \\
\hline $6 \mathbf{a}^{a)}$ & $3448,1694,1634$ & $2.15(2 \mathrm{H}$, quint.,$J=5.7 \mathrm{~Hz}), 3.78(2 \mathrm{H}, \mathrm{t}, J=5.7 \mathrm{~Hz}), 4.01(2 \mathrm{H}, \mathrm{t}, J=5.7 \mathrm{~Hz}), 7.72(1 \mathrm{H}, \mathrm{s}), 10.80(1 \mathrm{H}, \mathrm{br} \mathrm{s})$ \\
\hline $\mathbf{6 b}^{a)}$ & 3423,1682 & $1.89-2.16(4 \mathrm{H}, \mathrm{m}), 4.16(2 \mathrm{H}, \mathrm{t}, J=5.3 \mathrm{~Hz}), 4.30(2 \mathrm{H}, \mathrm{t}, J=5.3 \mathrm{~Hz}), 7.63(1 \mathrm{H}, \mathrm{s}), 10.93(1 \mathrm{H}, \mathrm{br} \mathrm{s})$ \\
\hline $6 c^{b)}$ & 1693,1664 & $\begin{array}{l}0.95(3 \mathrm{H}, \mathrm{t}, J=7.3 \mathrm{~Hz}), 1.67(2 \mathrm{H}, \text { sext., } J=7.3 \mathrm{~Hz}), 2.34(2 \mathrm{H}, \text { quint., } J=5.9 \mathrm{~Hz}), 3.97(2 \mathrm{H}, \mathrm{t}, J=7.3 \mathrm{~Hz}) \\
4.01(2 \mathrm{H}, \mathrm{t}, J=5.9 \mathrm{~Hz}), 4.18(2 \mathrm{H}, \mathrm{t}, J=5.9 \mathrm{~Hz}), 7.46(1 \mathrm{H}, \mathrm{s})\end{array}$ \\
\hline $\mathbf{6 d}^{b)}$ & 1697,1654 & $\begin{array}{l}0.95(3 \mathrm{H}, \mathrm{t}, J=7.3 \mathrm{~Hz}), 1.69(2 \mathrm{H}, \text { sext., } J=7.3 \mathrm{~Hz}), 2.04-2.27(4 \mathrm{H}, \mathrm{m}), 3.99(2 \mathrm{H}, \mathrm{t}, J=7.3 \mathrm{~Hz}), 4.25 \\
(2 \mathrm{H}, \mathrm{t}, J=5.4 \mathrm{~Hz}), 4.32(2 \mathrm{H}, \mathrm{t}, J=5.4 \mathrm{~Hz}), 7.36(1 \mathrm{H}, \mathrm{s}), 10.93(1 \mathrm{H}, \mathrm{br} \mathrm{s})\end{array}$ \\
\hline$d l-7 \mathbf{a}^{b)}$ & 1693,1651 & $\begin{array}{l}0.96(3 \mathrm{H}, \mathrm{t}, J=7.2 \mathrm{~Hz}), 0.97(3 \mathrm{H}, \mathrm{t}, J=7.2 \mathrm{~Hz}), 1.34(3 \mathrm{H}, \mathrm{d}, J=6.4 \mathrm{~Hz}), 1.52-2.04(4 \mathrm{H}, \mathrm{m}), 3.45 \\
(1 \mathrm{H}, \mathrm{dd}, J=7.1,9.1 \mathrm{~Hz}), 3.86-4.42(6 \mathrm{H}, \mathrm{m}), 7.43(1 \mathrm{H}, \mathrm{s})\end{array}$ \\
\hline$d l-7 \mathbf{b}^{a)}$ & $3423,1708,1664$ & $\begin{array}{l}0.87(3 \mathrm{H}, \mathrm{t}, J=7.3 \mathrm{~Hz}), 1.30(3 \mathrm{H}, \mathrm{d}, J=5.9 \mathrm{~Hz}), 1.68(2 \mathrm{H}, \text { sext., } J=7.3 \mathrm{~Hz}), 3.92(2 \mathrm{H}, \mathrm{t}, J=7.3 \mathrm{~Hz}) \text {, } \\
4.05-4.34(1 \mathrm{H}, \mathrm{m}), 7.60(1 \mathrm{H}, \mathrm{s}), 12.40(1 \mathrm{H}, \mathrm{br} \mathrm{s})\end{array}$ \\
\hline$d l-7 \mathbf{c b}^{b)}$ & 1685,1653 & $\begin{array}{l}0.94(3 \mathrm{H}, \mathrm{t}, J=7.3 \mathrm{~Hz}), 0.97(3 \mathrm{H}, \mathrm{t}, J=7.2 \mathrm{~Hz}), 1.34(3 \mathrm{H}, \mathrm{d}, J=6.4 \mathrm{~Hz}), 1.60-2.04(4 \mathrm{H}, \mathrm{m}), 3.45 \\
(1 \mathrm{H}, \mathrm{dd}, J=6.8,10.4 \mathrm{~Hz}), 3.89-4.49(6 \mathrm{H}, \mathrm{m}), 7.52(1 \mathrm{H}, \mathrm{s})\end{array}$ \\
\hline$d l-8 \mathbf{a}^{b)}$ & 1691,1658 & $\begin{array}{l}0.96(6 \mathrm{H}, \mathrm{t}, J=7.4 \mathrm{~Hz}), 1.44(3 \mathrm{H}, \mathrm{d}, J=5.9 \mathrm{~Hz}), 1.64-2.05(4 \mathrm{H}, \mathrm{m}), 3.64(1 \mathrm{H}, \mathrm{dd}, J=4.6,13.7 \mathrm{~Hz}) \\
3.88-4.48(6 \mathrm{H}, \mathrm{m}), 7.45(1 \mathrm{H}, \mathrm{s})\end{array}$ \\
\hline$d l-\mathbf{8} \mathbf{b}^{a)}$ & 1710,1654 & $\begin{array}{l}0.87(3 \mathrm{H}, \mathrm{t}, J=7.3 \mathrm{~Hz}), 1.38(3 \mathrm{H}, \mathrm{d}, J=6.1 \mathrm{~Hz}), 1.68(2 \mathrm{H}, \mathrm{sext} ., J=7.3 \mathrm{~Hz}), 4.02(2 \mathrm{H}, \mathrm{t}, J=7.3 \mathrm{~Hz}) \\
4.47-4.66(1 \mathrm{H}, \mathrm{m}), 7.69(1 \mathrm{H}, \mathrm{s}), 12.65(1 \mathrm{H}, \mathrm{br} \mathrm{s})\end{array}$ \\
\hline$d l-8 \mathbf{c}^{b)}$ & 1689,1658 & $\begin{array}{l}0.95(3 \mathrm{H}, \mathrm{t}, J=7.3 \mathrm{~Hz}), 0.97(3 \mathrm{H}, \mathrm{t}, J=7.2 \mathrm{~Hz}), 1.42(3 \mathrm{H}, \mathrm{d}, J=6.1 \mathrm{~Hz}), 1.50-2.12(4 \mathrm{H}, \mathrm{m}), 3.59 \\
(1 \mathrm{H}, \mathrm{dd}, J=4.4,13.7 \mathrm{~Hz}), 3.82-4.56(6 \mathrm{H}, \mathrm{m}), 7.42(1 \mathrm{H}, \mathrm{s})\end{array}$ \\
\hline
\end{tabular}

${ }^{1} \mathrm{H}-\mathrm{NMR}$ spectra were recorded in $a$ ) DMSO- $d_{6}$ or $b$ ) $\mathrm{CDCl}_{3}$.

Benzyl-2-(hydroxyproylamino)-1-propylpurin-6-one (19b) A mixture of $18(0.44 \mathrm{~g}, 1.5 \mathrm{mmol})$, aminoalcohol $(1 \mathrm{ml})$ and $\mathrm{CH}_{3} \mathrm{CN}(20 \mathrm{ml})$ were refluxed overnight. The reaction mixture was concentrated in vacuo and the residue was purified by recrystallization from $\mathrm{AcOEt}-\mathrm{MeOH}$ to give 19 .

19a: Yield: $52 \%$. mp $214-215^{\circ} \mathrm{C}$. ${ }^{1} \mathrm{H}-\mathrm{NMR}$ (DMSO- $d_{6}$ ) $\delta$ : $0.87(3 \mathrm{H}, \mathrm{t}$, $J=7.3 \mathrm{~Hz}), 1.55(2 \mathrm{H}$, sext., $J=7.3 \mathrm{~Hz}), 3.48(2 \mathrm{H}, \mathrm{t}, J=5.1 \mathrm{~Hz}), 3.90(2 \mathrm{H}, \mathrm{t}$, $J=7.3 \mathrm{~Hz}), 4.74(2 \mathrm{H}, \mathrm{t}, J=5.1 \mathrm{~Hz}), 5.44(2 \mathrm{H}, \mathrm{s}), 6.78(1 \mathrm{H}, \mathrm{br} \mathrm{s}), 7.30(5 \mathrm{H}$, s), $8.10(1 \mathrm{H}, \mathrm{s})$. IR $(\mathrm{KBr}) v_{\max }: 3340,1692 \mathrm{~cm}^{-1}$. Anal. Calcd for $\mathrm{C}_{17} \mathrm{H}_{21} \mathrm{~N}_{5} \mathrm{O}_{2}$ : C, 62.37; H, 6.47; N, 21.39. Found: C, 62.27; H, 6.65; N, 21.53 .

19b: Yield: 57\%. Oil. ${ }^{1} \mathrm{H}-\mathrm{NMR}\left(\mathrm{CDCl}_{3}\right) \delta: 0.92(3 \mathrm{H}, \mathrm{t}, J=7.3 \mathrm{~Hz})$, $1.48-2.04(4 \mathrm{H}, \mathrm{m}), 3.57-4.01(6 \mathrm{H}, \mathrm{m}), 5.48(2 \mathrm{H}, \mathrm{s}), 6.00(1 \mathrm{H}, \mathrm{br} \mathrm{s}), 7.30$ $(5 \mathrm{H}, \mathrm{s}), 7.62(1 \mathrm{H}, \mathrm{s})$. IR (KBr) $v_{\max }: 3356,1681 \mathrm{~cm}^{-1}$. HR-MS $m / z$ : 341.1854 (Calcd for $\mathrm{C}_{18} \mathrm{H}_{23} \mathrm{~N}_{5} \mathrm{O}_{2}: 341.1852$ ).

3-Benzyl-5-propyl-5,6,7,8-tetrahydro-3H-imidazo[2,1-b]purine-4-one (20a) and 3-Benzyl-5-propyl-5,6,7,8-tetrahydro-3H-pyrimido[2,1-b]purine-4-one (20b) To a solution of $\mathbf{1 9}(0.47 \mathrm{mmol})$ in $\mathrm{CH}_{2} \mathrm{Cl}_{2}(5 \mathrm{ml})$ was added $\mathrm{SOCl}_{2}(0.17 \mathrm{~g}, 1.4 \mathrm{mmol})$ at $0{ }^{\circ} \mathrm{C}$ and stirred for $12 \mathrm{~h}$ at rt. The reaction mixture was adjusted to $\mathrm{pH} 7-8$ with $4 \mathrm{M} \mathrm{NaOH}$. The $\mathrm{CH}_{2} \mathrm{Cl}_{2}$ layer was washed with brine, dried and concentrated. The residue was chromatographed on silica gel using $\mathrm{CHCl}_{3}-\mathrm{MeOH}(10: 1)$ as an eluent to give $\mathbf{2 0}$, which was recrystallized from iso-propylether.

20a: Yield: $67 \%$. mp $133-134{ }^{\circ} \mathrm{C} .{ }^{1} \mathrm{H}-\mathrm{NMR}\left(\mathrm{CDCl}_{3}\right) \delta: 0.96(3 \mathrm{H}, \mathrm{t}, J=$ $7.5 \mathrm{~Hz}), 1.72(2 \mathrm{H}$, sext., $J=7.5 \mathrm{~Hz}), 3.75-4.16(6 \mathrm{H}, \mathrm{m}), 5.44(2 \mathrm{H}, \mathrm{s}), 7.34$ $(5 \mathrm{H}, \mathrm{s}), 7.40(1 \mathrm{H}, \mathrm{s})$. IR $(\mathrm{KBr}) v_{\max }: 1686,1642 \mathrm{~cm}^{-1}$. Anal. Calcd for $\mathrm{C}_{17} \mathrm{H}_{19} \mathrm{~N}_{5} \mathrm{O}: \mathrm{C}, 66.00 ; \mathrm{H}, 6.19 ; \mathrm{N}, 22.64$. Found: C, 65.95; H, 6.27; N, 22.58.

20b: Yield: $72 \%$. mp $160-161^{\circ} \mathrm{C} .{ }^{1} \mathrm{H}-\mathrm{NMR}\left(\mathrm{CDCl}_{3}\right) \delta: 0.92(3 \mathrm{H}, \mathrm{t}$, $J=7.3 \mathrm{~Hz}), 1.74(2 \mathrm{H}$, sext., $J=7.3 \mathrm{~Hz}), 1.91(2 \mathrm{H}$, quint., $J=5.7 \mathrm{~Hz}), 3.56$ $(2 \mathrm{H}, \mathrm{t}, J=5.7 \mathrm{~Hz}), 3.95(2 \mathrm{H}, \mathrm{t}, J=7.3 \mathrm{~Hz}), 3.97(2 \mathrm{H}, \mathrm{t}, J=5.7 \mathrm{~Hz}), 5.46(2 \mathrm{H}$, $\mathrm{s}), 7.33(5 \mathrm{H}, \mathrm{s}), 7.39(1 \mathrm{H}, \mathrm{s})$. IR $(\mathrm{KBr}) v_{\max }: 1684,1634 \mathrm{~cm}^{-1}$. Anal. Calcd for $\mathrm{C}_{18} \mathrm{H}_{21} \mathrm{~N}_{5} \mathrm{O}: \mathrm{C}, 66.85 ; \mathrm{H}, 6.55 ; \mathrm{N}, 21.66$. Found: $\mathrm{C}, 66.70 ; \mathrm{H}, 6.64 ; \mathrm{N}$, 21.48 .

Propyl-5,6,7,8-tetrahydro-3H-imidazo[2,1-b]purine-4-one (5a) and 5Propyl-5,6,7,8-tetrahydro-3H-pyrimido[2,1-b]purine-4-one (5b) A mix- ture of $20(0.81 \mathrm{mmol})$ and $20 \%$ palladium hydroxide on carbon $(0.25 \mathrm{~g})$ in $\mathrm{MeOH}(30 \mathrm{ml})$ was shaken under hydrogen $(3$ atom) for $24 \mathrm{~h}$. The catalyst was removed and the filtrate was concentrated in vacuo, and the residue was recrystallized from $\mathrm{AcOEt}-\mathrm{MeOH}$ to yield $\mathbf{5}$.

3,5-Dipropyl-5,6,7,8-tetrahydro-3H-imidazo[2,1-b]purine-4-one (5c) and 3,5-Dipropyl-5,6,7,8-tetrahydro-3 $\mathrm{H}$-pyrimido[2,1-b]purine-4-one (5d) To a mixture of $5 \mathbf{a}$ or $\mathbf{5 b}(1.7 \mathrm{mmol})$ and anhydrous $\mathrm{K}_{2} \mathrm{CO}_{3}(0.35 \mathrm{~g}$, $2.5 \mathrm{mmol})$ in DMF $(10 \mathrm{ml})$ was added propyl bromide $(0.31 \mathrm{~g}, 2.5 \mathrm{mmol})$ and the mixture was stirred at $\mathrm{rt}$ for $10 \mathrm{~h}$, then concentrated in vacuo. The residue was chromatographed on silica gel using $\mathrm{CHCl}_{3}-\mathrm{MeOH}(20: 1)$ as an eluent to give $\mathbf{5 c}$ or $\mathbf{5 d}$, which was recrystallized from petrolum ether.

4,5-Dihydro-9H-pyrimido[1,2,3-c,d]purine-8,10-dione (6a) and 4,5-Dihydro-10H-diazepino[1,2,3-c, $d]$ purine-9,11-dione (6b) A suspension of $21(4.6 \mathrm{mmol})$ in $\mathrm{H}_{2} \mathrm{O}(100 \mathrm{ml})$ was hydrogenated over $10 \%$ palladium on carbon $(0.15 \mathrm{~g})$ at 3 atom for $15 \mathrm{~h}$. The catalyst was removed and the filtrate was concentrated in vacuo. A mixture of the residue, DMF $(20 \mathrm{ml})$, triethyl orthoformate $(1.1 \mathrm{~g}, 7.5 \mathrm{mmol})$ and $p$-toluenesulfonic acid $(0.050 \mathrm{~g})$ was stirred overnight at $100^{\circ} \mathrm{C}$. The reaction mixture was then concentrated in vacuo and the residue was chromatographed on silica gel using $\mathrm{CHCl}_{3}-$ $\mathrm{MeOH}(3: 1)$ as an eluent to give 6 , which was recrystallized from EtOH.

9-Propyl-4,5-dihydro-9H-pyrimido[1,2,3-c,d]purine-8,10-dione (6c) and 10-Propyl-4,5-dihydro-10H-diazepino[1,2,3-c, $d]$ purine-9,11-dione (6d) A mixture of $6 \mathbf{a}$ or $6 \mathbf{b}(6.0 \mathrm{mmol})$ and anhydrous $\mathrm{K}_{2} \mathrm{CO}_{3}(1.5 \mathrm{~g}, 12$ mmol) and propyl bromide $(1.6 \mathrm{~g}, 12 \mathrm{mmol})$ in DMF $(20 \mathrm{ml})$ was stirred overnight at $60^{\circ} \mathrm{C}$. The reaction mixture was concentrated in vacuo and the residue was chromatographed on silica gel using $\mathrm{CHCl}_{3}-\mathrm{MeOH}(10: 1)$ as an eluent to give $\mathbf{6 c}$ or $\mathbf{6 d}$, which was recrystallized from AcOEt-MeOH.

dl-6-[(2-Hydroxy-1-methyl)ethylamino]-3,7-dipropylpurin-2-one (dl24) To $23(0.51 \mathrm{~g}, 2.0 \mathrm{mmol})$ in pyridine $(10 \mathrm{ml})$ was added $d l$-2-amino-1propanol $(4 \mathrm{ml})$ and the mixture was refluxed overnight. The reaction mixture was evaporated in vacuo and the residue was chromatographed on silica gel using $\mathrm{CHCl}_{3}-\mathrm{MeOH}(3: 1)$ as an eluent to give $d l-24(0.39 \mathrm{~g}, 83 \%) .{ }^{1} \mathrm{H}-$ $\operatorname{NMR}\left(\mathrm{CDCl}_{3}\right) \delta: 0.95(3 \mathrm{H}, \mathrm{t}, J=7.3 \mathrm{~Hz}), 0.98(3 \mathrm{H}, \mathrm{t}, J=7.3 \mathrm{~Hz}), 1.27(3 \mathrm{H}$, d, $J=6.6 \mathrm{~Hz}), 1.64-2.03(4 \mathrm{H}, \mathrm{m}), 2.45(1 \mathrm{H}, \mathrm{br} \mathrm{s}), 3.50-4.28(7 \mathrm{H}, \mathrm{m}), 4.49$ 
$(1 \mathrm{H}, \mathrm{brs}), 7.47(1 \mathrm{H}, \mathrm{s})$. IR (KBr) $v_{\max }: 3390,1633 \mathrm{~cm}^{-1}$. HR-MS $m / z$ : 293.1857 (Calcd for $\mathrm{C}_{14} \mathrm{H}_{23} \mathrm{~N}_{5} \mathrm{O}_{2}: 293.1852$ ).

Compounds $d l-\mathbf{2 5}, d l-027$ and $d l-28$ were prepared by a similar procedure to that described above from $\mathbf{2 3}$ or $\mathbf{2 6} .{ }^{9)}$

$d l$-6-[(2-Hydroxy-2-methyl)ethylamino]-3,7-dipropylpurin-2-one (dl-25): Yield: $72 \% .{ }^{1} \mathrm{H}-\mathrm{NMR}\left(\mathrm{CDCl}_{3}\right) \delta: 0.95(6 \mathrm{H}, \mathrm{t}, J=7.1 \mathrm{~Hz}), 1.18(3 \mathrm{H}, \mathrm{d}, J=6.2$ $\mathrm{Hz}), 1.64-2.03(4 \mathrm{H}, \mathrm{m}), 2.70(1 \mathrm{H}, \mathrm{br} \mathrm{s}), 3.20-3.48(2 \mathrm{H}, \mathrm{m}), 3.73-4.31$ $(5 \mathrm{H}, \mathrm{m}), 6.07(1 \mathrm{H}, \mathrm{brs}), 7.48(1 \mathrm{H}, \mathrm{s}) . \mathrm{IR}(\mathrm{KBr}) v_{\max }: 3323,1628$, $1610 \mathrm{~cm}^{-1}$. HR-MS m/z: 293.1856 (Calcd for $\mathrm{C}_{14} \mathrm{H}_{23} \mathrm{~N}_{5} \mathrm{O}_{2}: 293.1852$ ).

dl-6-[(2-Hydroxy-1-methyl)ethylamino]-3-propylpurin-2-one ( $d l-27)$ : Yield: $85 \%$. mp $296-297^{\circ} \mathrm{C}$. ${ }^{1} \mathrm{H}-\mathrm{NMR}$ (DMSO- $\left.d_{6}\right) \delta$ : $0.94(3 \mathrm{H}, \mathrm{t}, J=7.3$ $\mathrm{Hz}), 1.17(3 \mathrm{H}, \mathrm{d}, J=6.8 \mathrm{~Hz}), 1.62(2 \mathrm{H}$, sext., $J=7.3 \mathrm{~Hz}), 3.88(2 \mathrm{H}, \mathrm{t}, J=7.3$ $\mathrm{Hz}), 4.23(1 \mathrm{H}$, br s), $4.95(1 \mathrm{H}$, br s), $7.23(1 \mathrm{H}, \mathrm{brs}) 7.88(1 \mathrm{H}, \mathrm{s})$. IR $(\mathrm{KBr})$ $v_{\max }: 3325,1635,1612 \mathrm{~cm}^{-1}$. Anal. Calcd for $\mathrm{C}_{11} \mathrm{H}_{17} \mathrm{~N}_{5} \mathrm{O}_{2}: \mathrm{C}, 52.58 ; \mathrm{H}$, $6.82 ; \mathrm{N}, 27.87$. Found: C, 57.92; H, 6.34; N, 22.56.

dl-6-[(2-Hydroxy-2-methyl)ethylamino]-3-propylpurin-2-one (dl-28): Yield: $81 \%$. mp $288-289^{\circ} \mathrm{C}$. ${ }^{1} \mathrm{H}-\mathrm{NMR}$ (DMSO- $\left.d_{6}\right) \delta: 0.85(3 \mathrm{H}, \mathrm{t}, J=7.3$ $\mathrm{Hz}), 1.10(3 \mathrm{H}, \mathrm{d}, J=6.2 \mathrm{~Hz}), 1.62(2 \mathrm{H}$, sext., $J=7.3 \mathrm{~Hz}), 3.87(2 \mathrm{H}, \mathrm{t}, J=7.3$ $\mathrm{Hz}), 5.10(1 \mathrm{H}, \mathrm{br} \mathrm{s}), 7.60(1 \mathrm{H}, \mathrm{br} \mathrm{s}) 7.88(1 \mathrm{H}, \mathrm{s})$. IR $(\mathrm{KBr}) v_{\max }: 3448,3309$, $3238,1643,1614 \mathrm{~cm}^{-1}$. Anal. Calcd for $\mathrm{C}_{11} \mathrm{H}_{17} \mathrm{~N}_{5} \mathrm{O}_{2}: \mathrm{C}, 52.58 ; \mathrm{H}, 6.82 ; \mathrm{N}$, 27.87. Found: C, $57.92 ; \mathrm{H}, 6.34 ; \mathrm{N}, 22.56$.

dl-1,4-Dipropyl-8-methyl-4,5,7,8-tetrahydro-3H-imidazo[2,1-i]purin5-one ( $d l-7$ a), $d l$-1,4-Dipropyl-7-methyl-4,5,7,8-tetrahydro-3H-imidazo[2,1-i]purin-5-one (dl-8a), dl-4-Propyl-8-methyl-4,5,7,8-tetrahydro-3Himidazo[2,1-i]purin-5-one (dl-7b), and $d l-4-$ Propyl-7-methyl-4,5,7,8tetrahydro-3H-imidazo[2,1-i]purin-5-one $(d l-8 b)$ To a mixture of $d l-24$, $d l-25, d l-27$ or $d l-28(1.3 \mathrm{mmol})$ and triethylamine $(0.16 \mathrm{~g}, 1.6 \mathrm{mmol})$ was added methanesulfonyl chloride $(0.18 \mathrm{~g}, 1.6 \mathrm{mmol})$ at $0^{\circ} \mathrm{C}$. The reaction mixture was stirred for $6 \mathrm{~h}$ at $\mathrm{rt}$, and then concentrated in vacuo. The residue was chromatographed on silica gel using $\mathrm{CHCl}_{3}-\mathrm{MeOH}(6: 1)$ as an eluent to give $d l-\mathbf{7} \mathbf{a}, d l-8 \mathbf{a}, d l-\mathbf{7 b}$ or $d l-\mathbf{8 b}$, which was recrystallized from AcOEt or petroleum ether.

$d l$-3,4-Dipropyl-8-methyl-4,5,7,8-tetrahydro-3H-imidazo[2,1-i]purin5-one $(d l-7 c)$ and $d l-3,4-D i p r o p y l-7-m e t h y l-4,5,7,8$-tetrahydro-3H-imidazo[2,1-i $]$ purin-5-one $(\boldsymbol{d l}-\mathbf{- 8 c}) \quad$ Compounds $d l-7 \mathbf{c}$ and $d l-8 \mathrm{c}$ were prepared from $d l-7 \mathbf{b}$ and $d l \mathbf{- 8 b}$, respectively as described above for $\mathbf{4 c}$.

Acknowledgements This study was partly supported by the Special Research Fund of Hokuriku University.

\section{References and Notes}

1) Ashton M. J., Cook D. C., Fenton G., Karlsson J.-A., Palfreyman M. N., Raeburn D., Ratcliffe A. J., Souness J. E., Thurairatnam S., Vicker
N., J. Med. Chem., 37, 1696-1703 (1994).

2) Raeburn D., Souness J. E., Tomkinson A., Karlsson J.-A., Prog. Drug. Res., 40, 9-32 (1993).

3) Miyamoto K., Sakai R., Kurita M., Ohmae S., Sanae F., Sawanishi H., Hasegawa T., Takagi K., Biol. Pharm. Bull., 18, $431-434$ (1995).

4) Miyamoto K., Kurita M., Ohmae S., Sanae F., Takagi K., Eur. J. Pharmacol. (Mol. Pharmacol. Soc.), 267, 317-322 (1994).

5) Sakai R., Konno K., Yamamoto Y., Sanae F., Takagi K., Hasegawa T., Iwasaki N., Kakiuchi, M., Kato H., Miyamoto K., J. Med. Chem., 35, 4039-4044 (1992).

6) Waki Y., Horita T., Miyamoto K., Ohya K., Kasugai S., Jpn. J. Pharmacol., 79, 477-483 (1999).

7) Miyamoto K., Waki Y., Horita T., Kasugai S., Biochem. Pharamcol., 54, 613-617 (1997).

8) Burnouf C., Pruniaux M.-P., Szilagy C. M., Annu. Rep. Med. Chem., 33, 91-109 (1998).

9) Sawanishi H., Suzuki H., Yamamoto S., Waki Y., Kasugai S., Ohya K., Suzuki N., Miyamoto K., Takagi K., J. Med. Chem., 40, 2—9 (1997).

10) Suzuki H., Sawanishi H., Yamamoto K., Yokogawa K., Miyamoto K., Chem. Pharm. Bull., 49, 188-191 (2001).

11) Suzuki H., Sawanishi H., Yamamoto K., Miyamoto K., Chem. Pharm. Bull., 47, 1322-1325 (1999).

12) Our investigation of the structure-activity relationships has shown that elongation of the alkyl group from ethyl to propyl at the 1-position of the xanthine skeleton markedly increased PDE4 inhibitory activity. ${ }^{3-5)}$ For this reasons, the propyl group as a substituent for the imidazole $N$ atom of the condensed purine was chosen.

13) Nagamatsu T., Yamasaki H., J. Chem. Soc., Chem. Commun., 1995, 2041-2043 (1995).

14) Ahn H.-S., Bercovici A., Boykow G., Bronnenkant A., Chackalamannil S., Chow J., Cleven R., Cook J., Czarniecki M., Domalski C., Fawzi A., Green M., Gundes A., Ho G., Laudicina M., Lindo N., Ma K., Mckittrick B., Mirzai B., Nechuta T., Neustadt T., Puchalski C., Pula K., Silverman L., Smith E., Stamfood A., Tedesco R. P., Tsai H., Tulshian D., Vaccaro H., Watkins R. W., Weng X., Witkowski J. T., Xia Y., Zhang H., J. Med. Chem., 40, 2196-2210 (1997).

15) Fenn M. D., Lister J. H., Aust. J. Chem., 33, 1611-1617 (1980).

16) Muller C. E., Synthesis, 125-128 (1993).

17) Simo O., Rybar A., Alfoldi J., Synthesis, 835-840 (1995).

18) Uhlmann E., Prleiderer W., Heterocycles, 15, 437-453 (1981).

19) Catherine B., Marie P. P., Corinne M. S., Ann. Rep. Med. Chem., 33, 91-109 (1998). 